\title{
Decreased Expression of Angiotensin-Converting Enzyme 2 in Pancreatic Ductal Adenocarcinoma Is Associated with Tumor Progression
}

\author{
Lin Zhou, ${ }^{1, *}$ Ruifeng Zhang, ${ }^{2, *}$ WeiYan Yao, ${ }^{1}$ Jiancheng Wang, ${ }^{3}$ Aihua Qian, ${ }^{1}$ \\ Minmin Qiao, ${ }^{1}$ YongPing Zhang ${ }^{1}$ and YaOzong Yuan ${ }^{1}$ \\ ${ }^{1}$ Department of Gastroenterology, Ruijin Hospital, Shanghai Jiaotong University School of Medicine, Shanghai, \\ China \\ ${ }^{2}$ Respiratory Medicine, Ruijin Hospital, Shanghai Jiaotong University School of Medicine, Shanghai, China \\ ${ }^{3}$ General Surgery, Ruijin Hospital, Shanghai Jiaotong University School of Medicine, Shanghai, China
}

Angiotensin II (ANG II), the biologically active peptide of the renin-angiotensin system (RAS), is generated by angiotensin-converting enzyme (ACE) and is a regulator of cardiovascular homeostasis. Recently, there has been increasing evidence that ANG II is involved in the regulation of cell proliferation and migration, as well as angiogenesis via the ANG II-type 1 receptor (AT1R). These findings suggest that the ACE-ANG IIAT1R pathway is related to cancer biology. Previous reports have shown that ACE is preferentially expressed in pancreatic ductal adenocarcinoma (PDAC) tissues. Recently a homologue of ACE, angiotensin-converting enzyme 2 (ACE2), was reported to counterbalance the function of ACE, but the expression and role of ACE2 in PDAC are still unclear. In the present study, we analyzed the expression of ACE2 in invasive human PDAC and surrounding non-malignant tissues by Western blot analysis and immunohistochemistry. The ANG II concentration in homogenates of pancreatic tissues was measured with ELISA, and ACE2 protein was detected by Western blot analysis in BXPC3 and SW1990 human pancreatic ductal cancer cells. We have shown for the first time that the expression of ACE2 is decreased in PDAC tissues, in which ANG II was accumulated. Treatment of BxPC3 and SW1990 cells with ANG II decreased the expression of ACE2. Therefore, ANG II may contribute to the down-regulation of ACE2. Moreover, reduction of ACE2 expression by RNA interference promoted the proliferation of cultured pancreatic cancer cells. These findings suggest that ACE2 may have clinical potential as a novel molecular target for the treatment of PDAC. - angiotensin-converting enzyme 2; pancreatic ductal adenocarcinoma; angiotensin II; proliferation; angiotensin converting enzyme.

Tohoku J. Exp. Med., 2009, 217 (2), 123-131. (C) 2009 Tohoku University Medical Press

Pancreatic ductal adenocarcinoma (PDAC) is among the leading causes of cancer-related deaths worldwide (Sohn et al. 2000; Jemal et al. 2005; Jemal et al. 2008). Because of its insidious nature and anatomic location, PDAC is difficult to detect early, and the disease is often advanced by the time a diagnosis is established. In 2007, the number of new pancreatic cancer cases in the US was 37,170 and the number of deaths was 33,370 (Jemal et al. 2007). There is an urgent need for an improved understanding of molecular mechanisms that contribute to the aggressive nature of PDAC and for the design of novel effective therapies.

The renin-angiotensin system (RAS), consisting of renin, angiotensinogen, angiotensin I (ANG I), angiotensin II(ANG II), angiotensin-converting enzyme (ACE), and chymase, has a role in the autocrine and paracrine regula- tion of vascular function (Sugimoto et al. 2006). It is a cascade which results in the conversion of the inactive pro-hormone, ANG I, to the active peptide hormone ANG II by ACE. ANG II, the biologically active peptide of the RAS, is a major regulator of blood pressure and cardiovascular homeostasis, and is also recognized as a potent mitogen (Deshayes and Nahmias 2005). Recently, it has been shown that ANG II is involved in the regulation of cell proliferation, migration, inflammation, and tissue remodeling, as well as angiogenesis via the ANG II type 1 receptor (AT1R), (Greco et al. 2003; Uemura et al. 2003). These findings have suggested that the ACE-ANG II-AT1R pathway might be related to cancer biology (Deshayes and Nahmias 2005; $\mathrm{Neo}$ et al. 2007). The relationship between the RAS and PDAC was thus established. Recent studies have revealed

Received October 31, 2008; revision accepted for publication January 11, 2009.

* These two authors equally contributed to this work

Correspondence: Dr. Yaozong Yuan. Department of Gastroenterology, Ruijin Hospital, Shanghai Jiaotong University School of Medicine, Shanghai, 200025, China.

e-mail: yyz28@medmail.com.cn 
the local expression of many components of the RAS in various cancer cells and tissues, including pancreatic cancers (Deshayes and Nahmias 2005). ACE and AT1R are overexpressed in the majority (approximately 75\%) of PDAC patients. ACE is preferentially expressed in PDAC (Arafat et al. 2007), and ACE exerts an important role in the occurrence of PDAC through the formation of ANG II. Evidence for the involvement of the ACE-ANG II-AT1R pathway in the pathogenesis of PDAC, such as growth, metastasis, and angiogenesis, has accumulated in recent reports (Egami et al. 2003; Arrieta et al. 2005).

As a recently reported homologue of $\mathrm{ACE}$, angiotensin converting enzyme 2 (ACE2) is a new component of the updated RAS (Koka et al. 2008). This enzyme is highly expressed in the endothelial cells of the heart, kidney, and testis, and mainly degrades ANG II to angiotensin 1-7 (ANG 1-7), which is thought to act as a vasodilator and is involved in apoptosis and growth arrest, in contrast to ACE activity which generates ANG II and degrades ANG 1-9 (Epelman et al. 2008; Moon et al. 2008; Soler et al. 2008). Thus, ACE2 might be a potent counter-regulator against ACE and thus play a protective role in many diseases. However, the expression and role of ACE2 in PDAC is still unclear. In the present study, we have demonstrated for the first time that ACE2 is sparsely expressed in PDAC tissues, and the accumulated ANG II contributes to the downregulation of ACE2. Moreover, we have shown that reduction of ACE2 expression by RNA interference could promote the proliferation of pancreatic cancer cells in vitro. These discoveries have shed new light into understanding the mechanisms regulating the local RAS in the development and progression of PDAC. This key member of the RAS, ACE2, may therefore have enormous clinical potential as a novel molecular target for the treatment of PDAC.

\section{Materials ANd Methods}

\section{Patients and Specimens}

Tissue specimens of 15 PDAC patients ( 9 males and 6 females; age range, 43-77 years) were studied. All of the patients underwent pancreatectomy in the Department of Surgery of Ruijin Hospital of Shanghai Jiaotong University School of Medicine between April 2007 and September 2007. Tissue specimens were used for Western blotting and ANGII assays. In addition, formalin-fixed paraffin-embedded tissue blocks containing 80 PDAC and 35 adjacent tumor-free (TF) tissues, obtained from patients who underwent pancreatectomy between 2001 and 2007, were subjected to immunohistochemistry. Clinical information was obtained by chart review, and all diagnoses were confirmed histologically. This work was done with the approval of the Ethics Committee of Ruijin Hospital of Shanghai Jiaotong University School of Medicine. Written informed consent was obtained from all participants.

\section{Protein Extraction}

Two tissue samples were obtained from each PDAC patient who underwent pancreatectomy (one from the tumor mass and the other from the adjacent TF region). All samples were obtained immediately after surgery and transported in liquid nitrogen until extraction of pro- tein. Tissues were homogenized in RIPA buffer with $50 \mathrm{mmol} / \mathrm{L}$ of Tris- $\mathrm{HCl}$ buffer ( $\mathrm{pH}$ 8.0) containing $1 \mathrm{X}$ complete protease inhibitor cocktail tablets (Roche). The pancreatic ductal cancer cell lines, BxPC3 and SW1990, were washed with PBS before lysing (2X SDS). The tissue and cell lysates were centrifuged at 40,000 $\mathrm{g}$ for $60 \mathrm{~min}$ at $4^{\circ} \mathrm{C}$, and the supernatant containing total protein was used in further tests. The soluble fraction was used for Western blot analysis. The protein concentration was determined using the Bradford method.

\section{Immunohistochemical staining}

Immunohistochemical staining of $3-\mu \mathrm{m}$ paraffin sections for ACE and ACE2 proteins was performed with a LSAB kit (DAKO, Marseille, France) using mouse anti-ACE monoclonal (1 : 50; Santa Cruz, Delaware, CA) and goat anti-ACE2 polyclonal (1 : 50; Santa Cruz) antibodies. The sections were incubated in 3, 3'-diaminobenzide tetrahydrochloride with $0.05 \% \mathrm{H}_{2} \mathrm{O}_{2}$ for $3 \mathrm{~min}$ and then counterstained with Mayer's hematoxylin. Scoring of the pancreatic cancer tissue was performed by a histopathologist, and at least 500 tumor cells were counted in 5 different visual fields for each cancerous specimen. The intensity of staining (graded: 0 , negative; 1 , weak; 2 , moderate; and 3, strong) and the percentage of cells with positive immunoreactivity were recorded. The total score for each cellular compartment was obtained as the product of intensity and the percentage of staining. Weak or negative cases were defined as having a score $<1$, and strong cases had a score $\geq 1$.

\section{Western blot analysis}

The protein lysates were loaded onto $8 \%$ SDS-PAGE, and electrophoretically transferred to nitrocellulose membranes (Bio-Rad, Hercules, CA, USA). The membranes were blocked with 5\% non-fat dry milk solution in TBS with $0.1 \%$ Tween-20 (TBS/T; pH 7.6) for 1 $\mathrm{h}$ at room temperature, and then incubated in primary antibody dissolved in blocking solution at $4^{\circ} \mathrm{C}$ overnight. The ACE and ACE2 proteins were detected by primary antibodies. Mouse anti- $\beta$-actin monoclonal antibody (Merck, Darmstadt, Germany) was used to confirm equal loading. After washing the membranes 3 times for 5 min in TBS/T, they were incubated with horseradish peroxidase (HRP)conjugated secondary antibody (Cell Signaling, Beverly, MA, USA) corresponding to the primary antibody in blocking buffer for $1 \mathrm{~h}$ at room temperature. After three washes, the proteins were detected by a luminol detection reagent (Santa Cruz), and developed on Kodak $\mathrm{X}$-ray film.

Preparation of the tissue homogenates and ANGII concentration detection

The pancreatic tissues, after washing and removing connective tissues, were weighed and cut into small pieces. Homogenates (10\% $\mathrm{w} / \mathrm{v}$ ) were prepared in the solution containing $50 \mathrm{mM}$ phosphate buffer (pH 7.3) and 0.5 mM EDTA. The ANGII concentration in the culture homogenate was detected by ELISA kits (R\&D Minneapolis, MN, USA) according to the manufacturer's instructions.

\section{Cell lines and cultures}

The pancreatic ductal cancer cell lines, BxPC3 and SW1990, were originally obtained from the American Type Culture Collection (Rockville, MD, USA). All cell lines were routinely maintained in DMEM medium (Invitrogen, Grand Island, New York, USA) with $10 \%$ fetal bovine serum (Invitrogen) in a humidified incubator at $37^{\circ} \mathrm{C}$ with an atmosphere of $5 \% \mathrm{CO}_{2}$. 


\section{SiRNA knockdown of ACE2 expression}

SiRNA was designed and transfected into cells as described elsewhere with some modifications. The sequence for siRNA targeting ACE2 was as follows: forward 5'-CCA UCU ACA GUA CUG GAA A dTdT-3', reverse 5'-UUU CCA GUA CUG UAG AUG G dTdT-3' (JieKai, Shanghai, China). As a control for ACE2-siRNA, we used a corresponding random siRNA sequence (forward 5'-UUC UCC GAA CGU GUC ACG U dtdt-3', reverse 5'-ACG UGA CAC GUU CGG AGA A dtdt-3'). Cells were treated with siRNA targeting ACE2 at a concentration of $100 \mathrm{nM}$. SiRNA transfection was performed with lipofectamine-2000 (Invitrogen).

\section{Cell growth assay}

Cell growth was assessed with a Cell Counting Kit-8 (Dojindo, Kumamoto, Japan). The PDAC cell lines, BxPC3 and SW1990, transfected with non-targeting or ACE2-targeting siRNA, were plated in 96-well microplates. The cells were incubated in serum-free medium for different lengths of time, as indicated. After addition of $10 \mu 1$ of the Cell Counting Kit- 8 reagent followed by 3 hours of incubation, the plates were read at $450 \mathrm{~nm}$ in a spectrophotometer (Tecan Safire2, USA). Experiments were carried out in triplicate and repeated at least three times.

\section{Statistical analysis}

Data are presented as the means \pm S.D. The Student's $t$-test was used to compare the difference between two different groups. Chisquared analyses were performed to test the significance of the difference between various pathologic parameters. The correlation between the ACE2 protein expression score and TNM stages of PDAC was analyzed by Spearman's rank correlation test. A $P$ value $<0.05$ was considered statistically significant.

\section{Results}

Localization and expression of ACE and ACE2 proteins in PDAC and adjacent TF tissues

As shown in Fig. 1, the cellular localization of ACE and ACE2 was similar, with both localized to the membrane and cytoplasm. ACE2 immunostainning was particularly strong in the normal ductal epithelial cells (Fig. 1B). Serial sections revealed a higher expression of ACE2 in TF than PDAC tissues (Fig. 1E). ACE immunostainning was moderate or negative in TF tissues (Fig. 1A); however, ACE was markedly increased, particularly in the ductal cancer cells in PDAC (Fig. 1C).

\section{Immunohistochemical analysis of ACE2 protein in PDAC tissues}

Among 80 samples of PDAC tissues, 31(38.75\%) strongly expressed ACE2 protein (score $\geq 1$ ). Serial sections of PDAC tissues demonstrated a marked decrease in ACE2 expression compared with ACE expression (Fig. 1E). We next analyzed the relationship between ACE2 protein expression and several clinicopathologic factors (Table 1). The strong staining of ACE2 was much higher in patients with TNM stages I+II than stages III+IV $(P<0.05)$. Moreover, ACE2 protein expression in PDAC tissues was negatively correlated with TNM stage $(r=-0.405, P<0.01$;
Fig. 1F). There were no significant correlation between the level of ACE2 expression and other clinicopathologic characteristics, such as age, gender, histologic differentiation, and metastasis.

\section{Expression of ACE and ACE2 in normal and PDAC tis- sues}

We carried out Western blotting analysis on the two proteins. As shown in Fig. 2, in all 15 pairs of specimens, ACE protein expression was higher in PDAC tissues than the adjacent TF tissues. However, ACE2 protein expression was lower in PDAC tissues than the adjacent TF tissues.

\section{Accumulated ANGII downregulated the expression of ACE2 protein in PDAC cell lines}

Previous studies have shown that ANG II inhibits the synthesis of ACE2 at the mRNA and protein levels. In our study, we found that the ANG II concentration in the tissue homogenate of PDAC was higher than the adjacent TF tissues (Fig. 3A). To investigate whether ANG II could downregulate the expression of ACE2 protein in pancreatic cancer cell lines, BxPC3 and SW1990 cells were treated with or without ANGII (1-10 $\mu \mathrm{mol} / \mathrm{L})$. Protein concentration analysis by Western blot showed that ANGII downregulated ACE2 in both cell lines (Fig. 3B).

\section{RNA interference-mediated reduction in ACE2 expres-} sion resulted in enhanced cell proliferation

RNA interference was effective in reducing ACE2 protein expression in BxPC3 and SW1990 cells (Fig. 4A). A Cell Counting Kit- 8 was used to assess the effects of ACE2 knockdown on cell proliferation. In the cell proliferation assay, we found that both cells transfected with ACE2targeting siRNA showed significantly higher WST-8 cleavage levels than controls (Fig. 4B). This study demonstrated that ACE2 inhibited the proliferation of pancreatic cancer cells.

\section{Discussion}

The RAS is a cascade which results in the conversion of the inactive pro-hormone, ANG I, to the active peptide hormone, ANG II, by ACE. The expression of many components of the RAS in various cancers, including cancers of the pancreas, brain, lung, breast, prostate, skin, and cervix, and glioblastoma, has been previously demonstrated (Arafat et al. 2007). In carcinogenesis, the ACE-ANG II-AT1R signaling pathway is associated with cell proliferation, angiogenesis, inflammation, and metastasis. These results indicate that ACE exerts an important role in the pathogenesis of PDAC by the formation of ANG II. As a recently reported homologue of ACE, ACE2 is a new component of the updated RAS. ACE2 has been shown to be a critical element in the conversion to hyperplasia via anti-apoptotic hormone [ANG II directly to ANG-(1-7)], which has the opposite function of ANG II (i.e., anti-proliferative and proapoptotic). ACE2 appears to counterbalance the ANG II- 

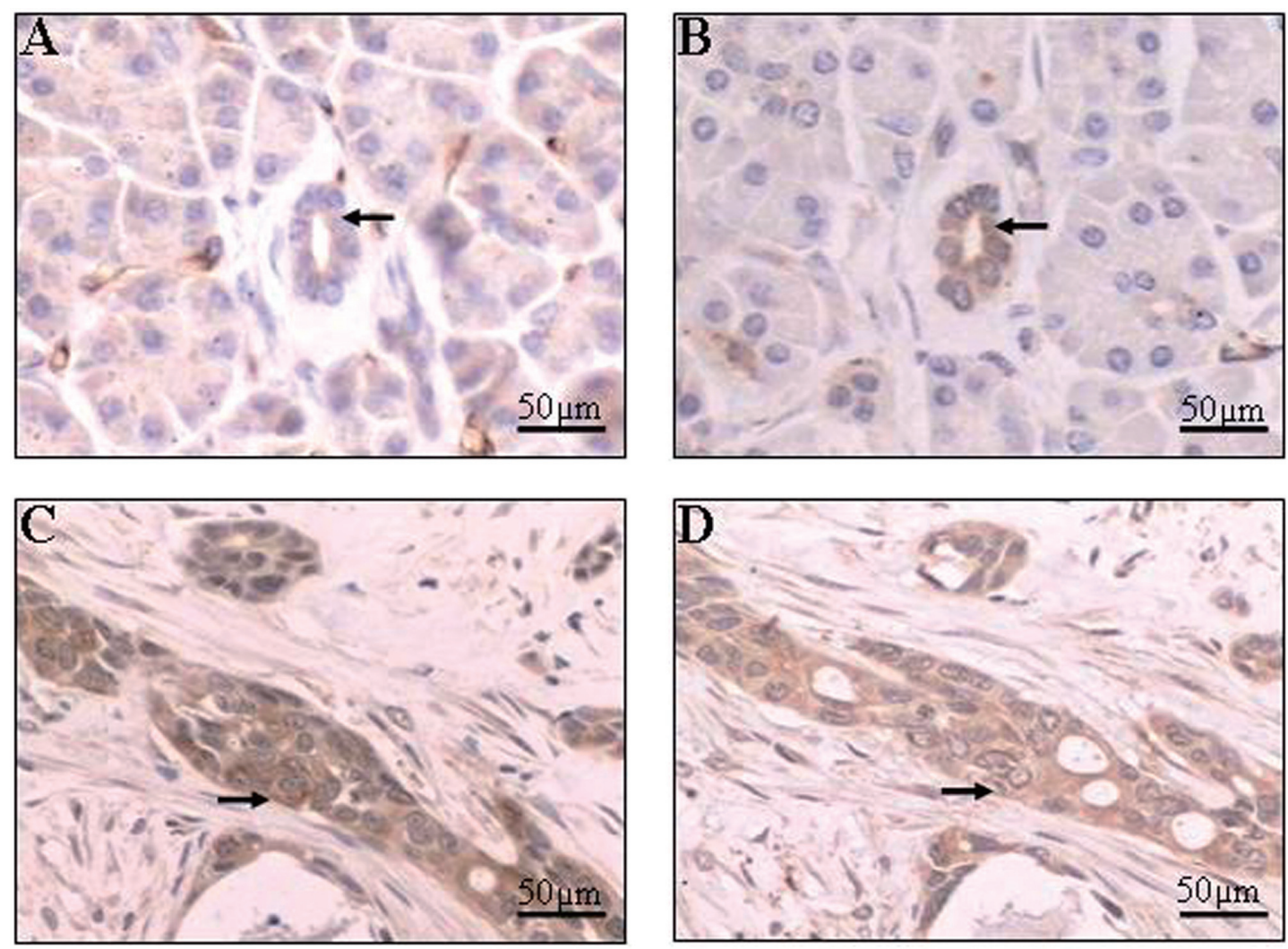

$\mathbf{E}$

$\mathbf{F}$
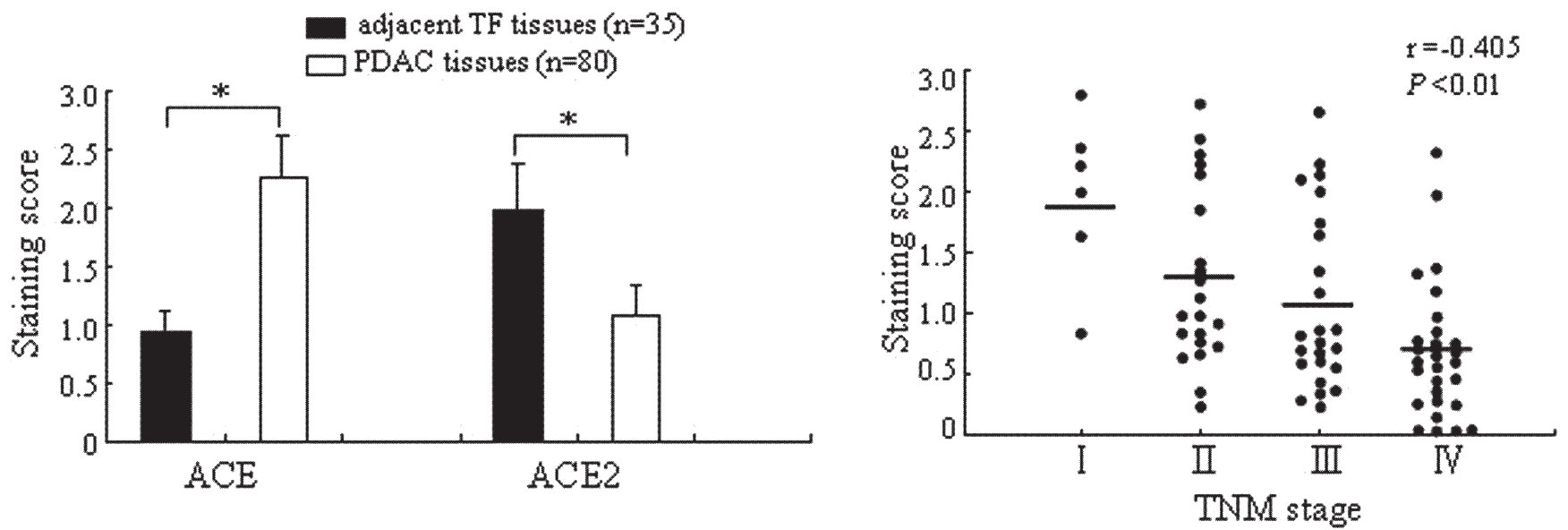

Fig. 1. Immunostaining for ACE and ACE2 in pancreatic tissues. Serial sections of adjacent TF tissues were stained with antibodies to ACE (A) and ACE2 (B). Serial sections of PDAC tissues were stained with antibodies to ACE (C) and ACE2 (D). Immunoreactivity (arrows) was detected in normal ductal cells (A, B) and ductal cancer cells (C, D). (E) Semiquantitative analysis of ACE and ACE2 immunohistochemical staining. $* P<0.05$. (F) ACE2 protein expression was significantly correlated with TNM stages of PDAC $(r=-0.405, P<0.01$, Spearman's rank correlation test).

promoting effect of ACE, and plays a protective role in many diseases (Raizada and Ferreira 2007; Mizuiri et al. 2008; Wang et al. 2008). However, the expression and role of ACE2 in PDAC are still unclear.

Consistent with a previous report (Arafat et al. 2007), the present study showed high levels of ACE in PDAC tis- sues. ANG II was mainly from ANG I catalyzed by ACE, and we also found the ANG II concentration was higher in the homogenate of PDAC tissues than adjacent TF tissues. However, our data showed that ACE2 protein expression was relatively lower in PDAC tissues than TF tissues by immunohistochemical and Western blot analysis. ANG II, 
TABLE 1. Statistical analysis of ACE2 protein expression and clinical characteristics of patients with PDAC.

\begin{tabular}{|c|c|c|c|c|}
\hline \multirow{2}{*}{ Characteristics } & \multirow{2}{*}{ Total } & \multicolumn{2}{|c|}{ ACE2 protein expression } & \multirow{2}{*}{$P$-value } \\
\hline & & Strong & Weak & \\
\hline \multicolumn{5}{|l|}{ Age at surgery(years) } \\
\hline$<55$ & 52 & 21 & 31 & \multirow{2}{*}{0.683} \\
\hline$\geq 55$ & 28 & 10 & 18 & \\
\hline \multicolumn{5}{|l|}{ Gender } \\
\hline Male & 59 & 23 & 36 & \multirow{2}{*}{0.943} \\
\hline Female & 21 & 8 & 13 & \\
\hline \multicolumn{5}{|l|}{ TNM stage } \\
\hline I + II & 27 & 16 & 11 & \multirow{2}{*}{0.007} \\
\hline $\mathrm{III}+\mathrm{IV}$ & 53 & 15 & 38 & \\
\hline \multicolumn{5}{|l|}{ Differentiation } \\
\hline Well & 19 & 8 & 11 & \multirow{3}{*}{0.835} \\
\hline Moderate & 32 & 13 & 19 & \\
\hline Poor & 29 & 10 & 19 & \\
\hline \multicolumn{5}{|c|}{ Lymph node metastasis } \\
\hline Yes & 35 & 14 & 21 & \multirow{2}{*}{0.840} \\
\hline No & 45 & 17 & 28 & \\
\hline \multicolumn{5}{|l|}{ Vascular invasion } \\
\hline Yes & 42 & 18 & 24 & \multirow{2}{*}{0.428} \\
\hline No & 38 & 13 & 25 & \\
\hline \multicolumn{5}{|c|}{ ACE protein expression } \\
\hline Strong & 67 & 22 & 45 & \multirow{2}{*}{0.014} \\
\hline Weak & 13 & 9 & 4 & \\
\hline
\end{tabular}

through its interactions with the AT1 receptor, has been demonstrated to contribute to the pathogenesis of many diseases, including PDAC (Deshayes and Nahmias 2005; Arafat et al. 2007). ACE inhibitors (ACEI) and angiotensin receptor blockers (ARB) can attenuate ANG II actions or reduce ANG II production. These two agents are effective in pancreatic cancer therapy and prevention (Egami et al. 2003; Lindberg et al. 2004). Indeed, these two agents inhibit the growth of pancreatic cancer cells in vitro (Arafat et al. 2007). In a retrospective study of 5207 patients in Scotland, the relative risks of incident and fatal cancers among the 1559 patients treated with ACEI were significantly reduced to 0.72 and 0.65 , respectively. The relative risk of lung cancer in patients treated with ACEI was 0.34, which was significantly reduced as compared with treatment with other antihypertensive drugs, in which the relative risk was 0.98 (Lever et al. 1998). Recent studies have also shown that the expression of a new component of the RAS, ACE2, is enhanced with ACEI and ARB treatment (Ocaranza et al. 2006; Igase et al. 2008), suggesting a possible role of ACE2 in PDAC.

To further study the role of ACE2 in the pathogenesis of PDAC, ACE2 expression was reduced in pancreatic cancer cell lines by RNA interference. We found that reduction of ACE2 expression was accompanied by a significantly enhanced proliferative ability of cells. These findings sug- gested that ACE2 inhibited the proliferation of pancreatic cancer cells. ACE2 may play a protective role in PDAC by inhibiting the proliferation of tumor cells. However, it should be emphasized that carcinogenesis of the pancreas is a complicated event in which many factors are involved. So, further experiments, ideally using animal models, will be required to determine whether ACE2 is important in the process of PDAC.

Most groups agree, however, that deletion of ACE2 causes significant potentiation of the effects of ANGII (Yamamoto et al. 2006; Epelman et al. 2008; Moon et al. 2008; Soler et al. 2008). Yamamoto and his colleagues recently reported that deletion of ACE2 accelerates pressure overload-induced cardiac dysfunction in mice. They hypothesized that ACE2 plays an important role in dampening the hypertrophic response that is mediated by ANG II because the concentration of cardiac ANG II and the activation of mitogen-activated protein kinase by ANG II are markedly increased in ACE2-deficient mice (Yamamoto et al. 2006). Thus, we presume the anti-proliferative effect of ACE2 in PDAC is partially mediated by opposing the function of ANG II.

What causes downregulation of ACE2 in PDAC tissues? With the exception of the increased ANG II concentration in PDAC tissue homogenates, we also found that ANG II significantly inhibited ACE2 protein expression in 

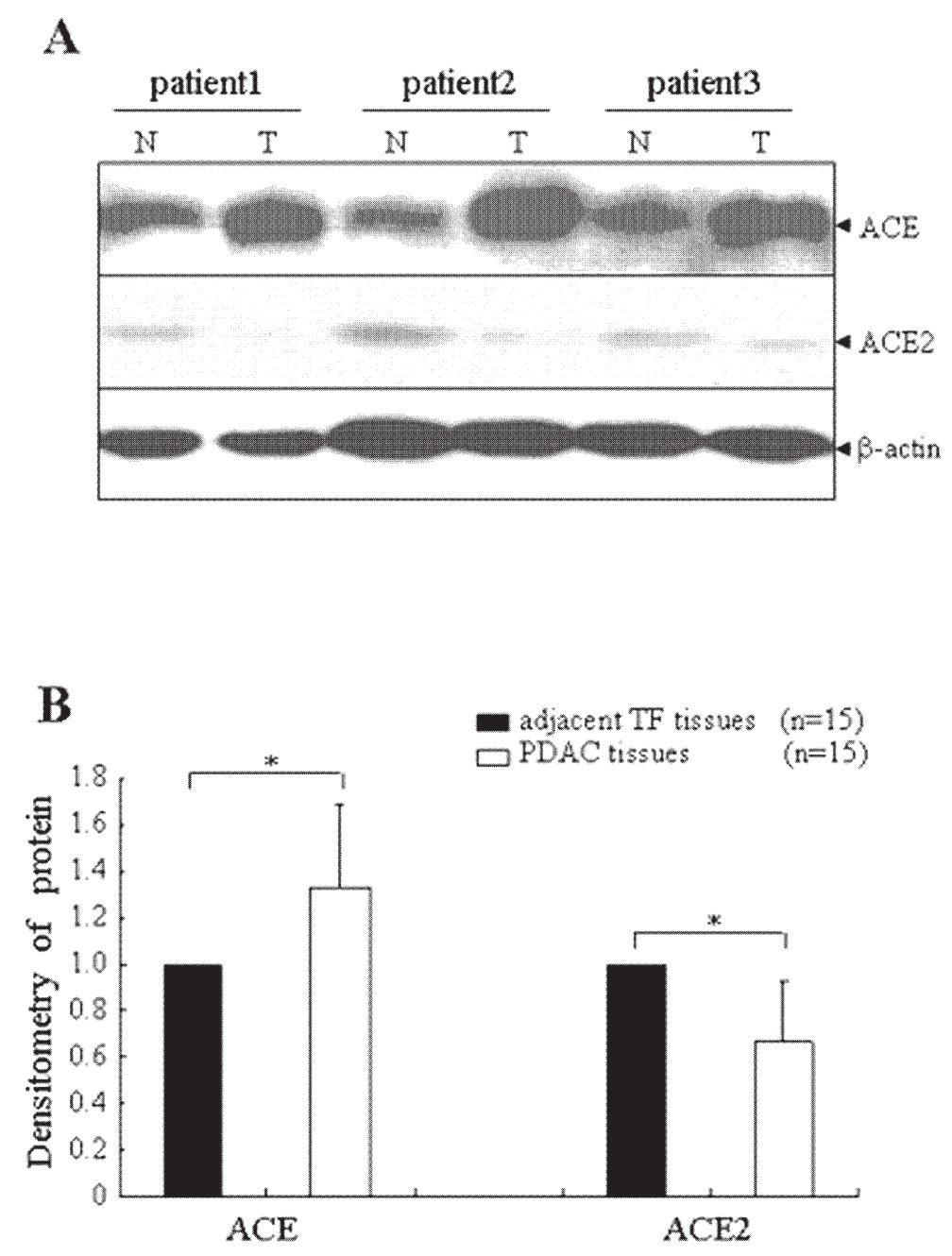

Fig. 2. Expression of ACE and ACE2 protein in human pancreatic tissues by Western blot. (A) Representative Western immunoblots of protein extracts from three adjacent TF and PDAC tissues showing expression of ACE and ACE2, with $\beta$-actin as an internal control. N represented adjacent TF tissue; T represented PDAC tissue. (B) Data in the graph represented the densitometric ratio of ACE and ACE2 to $\beta$-actin respectively. Average densitometry values of the samples were multiplied to obtain the arbitrary levels. The value in the panel represented as folds \pm S.D. $(n=15)$, with adjacent TF tissue taken as 1 . $* P<0.05$.

BxPC3 and SW1990 cells. The AT1 receptor-mediated ERK/p38 MAP kinase signaling pathway may be a key mechanism by which ANG II downregulates ACE2 expression (Koka et al. 2008). The downregulation of ACE2 by ANG II may serve as a mechanism within the pancreatic tissues to favor ANG II-mediated responses in PDAC.

ACEI and ARB are effective in the therapy and chemoprevention of cancers, including PDAC. Except blocking the production and formation of ANG II, the chemotherapeutic and chemopreventive effects may be due, at least in part, to an increase in ACE2. ACE2 is one of several enzymes that catalyzes the formation of degradation fragments, angiotensin 1-9 [ANG-(1-9)] and ANG-(1-7), from both ANG I and ANG II, respectively (Katovich et al. 2005). ACEI and ARB can attenuate ANG II actions or reduce ANG II formation. When these two agents are used, the expression of ACE2 is enhanced, which shifts the angio- tensin peptide balance favoring metabolism of ANG II to produce ANG-(1-7). In previous reports, the anti-proliferative responses to ANG-(1-7) are both dose- and timedependent (Campbell et al. 1993). ACEI and ARB can cause a significant elevation in both tissue and circulating ANG-(1-7) (Agata et al. 2006).

Many situations influence RAS activity, including taken ACEI or ARB, hypertension and renal disease. In the present study, tissue specimens for Western blotting were only from 15 PDAC patients. These 15 PDAC patients had not the history of hypertension or renal disease and did not take any ACE inhibitor or ARB. The tissue specimens for immunohistochemistry were from another 80 PDAC patients. In the 80 patients, 3 took ACE inhibitor or ARB in all 6 hypertension patients and no patients had renal disease. Only small portion of patients had the history of hypertension or renal disease and took ACEI or ARB. So it's hard to 


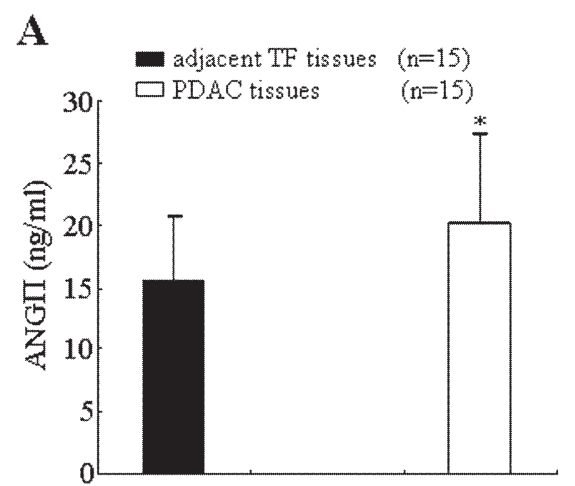

B

$\mathrm{BxPC} 3$

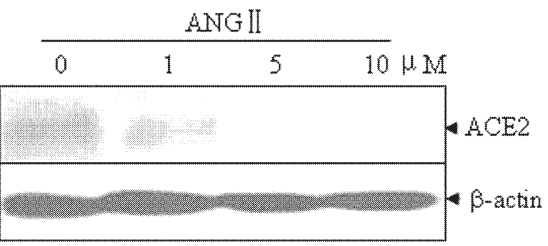

SW1990

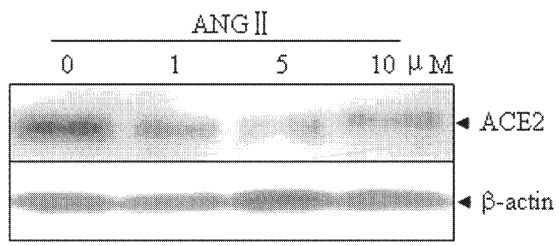

Fig. 3. ANGII downregulated ACE2 in PDAC cell lines. (A) The concentration of ANGII in pancreatic tissue homogenate was measured by ELISA. The value in panel represented means \pm S.D. $(n=15)$. $* P<0.05$. (B) Cells were incubated with $0,1,5$ or $10 \mu \mathrm{M}$ ANGII in serum-free media containing $0.5 \mathrm{mmol} / \mathrm{L}$ EDTA for $24 \mathrm{~h}$. ACE2 protein was detected by Western blot with $\beta$-actin as a loading control.

A

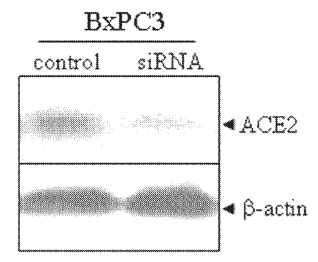

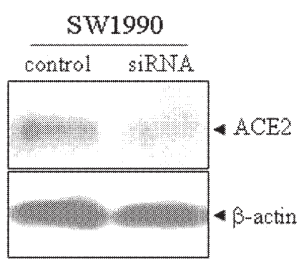
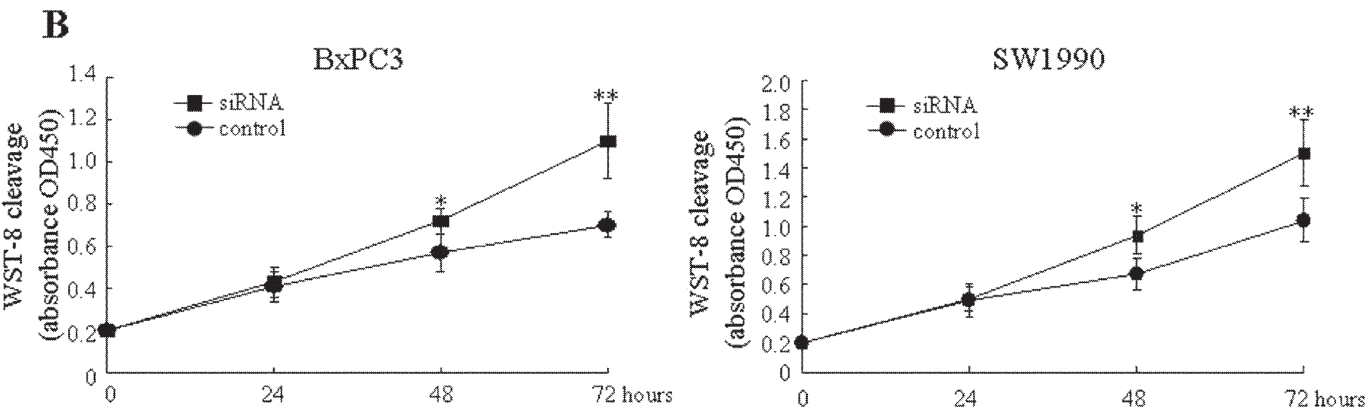

Fig. 4. RNA interference-mediated reduction in ACE2 expression resulted in enhanced cell proliferation ability. (A) BxPC3 and SW1990 were transiently transfected with siRNA targeting ACE2. Random siRNA was used as a control siRNA. ACE2 protein was detected by Western blot with $\beta$-actin as a loading control. (B) Effect of ACE2 knockdown on proliferation of cells. Cells transfected with non-targeting or ACE2-targeting siRNA were cultured for different hours. WST-8 cleavage in each group was measured (absorbance OD450). All results were represented as means \pm s.D. of three independent tests. $* P<0.05$ compared with control at the same time point.

make statistical analysis and judge whether these factors could affect ACE2 expression in PDAC. We will enlarge the number of tissue samples and investigate the changes of ACE2 when RAS activity is influenced, in our further study.

It is also noteworthy that the ratio of ACE/ACE2 is important. Recent research has shown that the imbalance of this ratio leads to many diseases (Brosnihan et al. 2005; Wakahara et al. 2007). Mice with targeted disruption of ACE2 develop abnormal heart function which progresses with age (Crackower et al. 2002). In our study, we showed 
that expression of ACE was higher in PDAC tissues; however, the ACE2 protein concentration was relatively lower in PDAC tissues. These results showed that the imbalance of the ACE/ACE2 ratio also exists in PDAC. It is reasonable to presume this imbalance may be involved in the pathogenesis of PDAC.

In summary, the results herein are the first demonstration that ACE2 is sparsely expressed in PDAC tissues, and the accumulated ANG II contributes to the downregulation of ACE2. Moreover, we showed that reduction of ACE2 expression by RNA interference promotes the proliferation of pancreatic cancer cells in vitro. These discoveries will be helpful in understanding the mechanisms by which the local RAS is regulated in the development and progression of PDAC. ACE2 may therefore have enormous clinical potential as a novel molecular target for the treatment of PDAC.

\section{Acknowledgment}

This work was supported by Key Program of Science and Technology Committee of Shanghai, China (06JC14055).

\section{References}

Agata, J., Ura, N., Yoshida, H., Shinshi, Y., Sasaki, H., Hyakkoku, M., Taniguchi, S. \& Shimamo, K. (2006) Olmesartan is an angiotensin II receptor blocker with an inhibitory effect on angiotensin-converting enzyme. Hypertens. Res., 29, 865-874.

Arafat, H.A., Gong, Q., Chipitsyna, G., Rizvi, A., Saa, C.T. \& Yeo, C.J. (2007) Antihypertensives as novel antineoplastics: Angiotensin-i-converting enzyme inhibitors and angiotensin II type 1 receptor blockers in pancreatic ductal adenocarcinoma. $J$. Am. Coll. Surg., 204, 996-1005.

Arrieta, O., Guevara, P. \& Escobar, E. (2005) Blockage of angiotensin II type I receptor decreases the synthesis of growth factors and induces apoptosis in C6 cultured cells and C6 rat glioma. Br. J. Cancer, 92, 1247-1252.

Brosnihan, K.B., Neves, L.A. \& Chappell, M.C. (2005) Does the angiotensin-converting enzyme (ACE)/ACE2 balance contribute to the fate of angiotensin peptides in programmed hypertension? Hypertension, 46, 1097-1099.

Campbell, D.J., Kladis, A. \& Duncan, A.M. (1993) Nephrectomy, converting enzyme inhibition, and angiotensin peptides. Hypertension, 22, 513-522.

Crackower, M.A., Sarao, R., Oudit, G.Y., Yagil, C., Kozieradzki, I., Scanga, S.E., Oliveira-dos-Santos, A.J., da Costa, J., Zhang, L., Pei, Y., Scholey, J., Ferrario, C.M., Manoukian, A.S., Chappell, M.C., Backx, P.H., Yagil, Y. \& Penninger, J.M. (2002) Angiotensin-converting enzyme 2 is an essential regulator of heart function. Nature, 417, 822-828.

Deshayes, F. \& Nahmias, C. (2005) Angiotensin receptors: A new role in cancer? Trend. Endocrinol. Metab., 16, 293-299.

Egami, K., Murohara, T., Shimada, T., Sasaki, K., Shintani, S., Sugaya, T., Ishii, M., Akagi, T., Ikeda, H., Matsuishi, T. \& Imaizumi, T. (2003) Role of host angiotensin II type 1 receptor in tumor angiogenesis and growth. J. Clin. Invest., 112, 67-75.

Epelman, S., Tang, W.H., Chen, S.Y., Van, Lente F., Francis, G.S. \& Sen, S. (2008) Detection of soluble angiotensin-converting enzyme 2 in heart failure: Insights into the endogenous counter-regulatory pathway of the renin-angiotensin-aldosterone system. J. Am. Coll. Cardiol., 52, 750-754.

Greco, S., Muscella, A., Elia, M.G., Salvatore, P., Storelli, C., Mazzotta, A., Manca, C. \& Marsigliante, S. (2003) Angiotensin II activates extracellular signal regulated kinases via pro- tein kinase $\mathrm{c}$ and epidermal growth factor receptor in breast cancer cells. J. Cell. Physiol., 196, 370-377.

Igase, M., Kohara, K., Nagai, T., Miki, T. \& Ferrario, C.M. (2008) Increased expression of angiotensin converting enzyme 2 in conjunction with reduction of neointima by angiotensin II type 1 receptor blockade. Hypertens. Res., 31, 553-559.

Jemal, A., Murray, T., Ward, E., Samuels, A., Tiwari, R.C., Ghafoor, A., Feuer, E.J. \& Thun, M.J. (2005) Cancer statistics, 2005. CA Cancer J. Clin., 55, 10-30.

Jemal, A., Siegel, R., Ward, E., Murray, T., Xu, J. \& Thun, M.J. (2007) Cancer statistics, 2007. CA Cancer J. Clin., 57, 43-66.

Jemal, A., Siegel, R., Ward, E., Hao, Y., Xu, J., Murray, T. \& Thun, M.J. (2008) Cancer statistics, 2008. CA Cancer J. Clin., 58, 71-96.

Katovich, M.J., Grobe, J.L., Huentelman, M. \& Raizada, M.K. (2005) Angiotensin-converting enzyme 2 as a novel target for gene therapy for hypertension. Exp. Physiol., 90, 299-305.

Koka, V., Huang, X.R., Chung, A.C., Wang, W., Truong, L.D. \& Lan, H.Y. (2008) Angiotensin ii up-regulates angiotensin i-converting enzyme (ACE), but down-regulates ACE2 via the at1-erk/p38 map kinase pathway. Am. J. Pathol., 172, $1174-1183$.

Lever, A.F., Hole, D.J., Gillis, C.R., McCallum, I.R.M.G.T., MacKinnon, P.L., Meredith, P.A., Murray, L.S., Reid, J.L. \& Robertson, M.J. (1998) Do inhibitors of angiotensin-I-converting enzyme protect against risk of cancer? Lancet, 352, 179-184.

Lindberg, H., Nielsen, D., Jensen, B.V., Eriksen, J. \& Skovsgaard, T. (2004) Angiotensin converting enzyme inhibitors for cancer treatment? Acta Oncol., 43, 142-152.

Mizuiri, S., Hemmi, H., Arita, M., Ohashi, Y., Tanaka, Y., Miyagi, M., Sakai, K., Ishikawa, Y., Shibuya, K., Hase, H. \& Aikawa, A. (2008) Expression of ACE and ACE2 in individuals with diabetic kidney disease and healthy controls. Am. J. Kidney Dis., 51, 613-623.

Moon, J.Y., Jeong, K.H., Lee, S.H., Lee, T.W., Ihm, C.G. \& Lim S.J. (2008) Renal ACE and ACE2 expression in early diabetic rats. Nephron Exp. Nephrol., 110, 8-16.

Neo, J.H., Malcontenti-Wilson, C., Muralidharan, V. \& Christophi C. (2007) Effect of ACE inhibitors and angiotensin II receptor antagonists in a mouse model of colorectal cancer liver metastases. J. Gastroenterol. Hepatol., 22, 577-584.

Ocaranza, M.P., Godoy, I., Jalil, J.E., Varas, M., Collantes, P., Pinto, M., Roman, M., Ramirez, C., Copaja, M., Diaz-Araya, G., Castro, P. \& Lavandero, S. (2006) Enalapril attenuates downregulation of angiotensin-converting enzyme 2 in the late phase of ventricular dysfunction in myocardial infarcted rat. Hypertension, 48, 572-578.

Raizada, M.K. \& Ferreira, A.J. (2007) ACE2: A new target for cardiovascular disease therapeutics. J. Cardiovasc. Pharmacol., 50, 112-119.

Sohn, T.A., Yeo, C.J., Cameron, J.L., Koniaris, L., Kaushal, S., Abrams, R.A., Sauter, P.K., Coleman, J., Hruban, R.H. \& Lillemoe, K.D. (2000) Resected adenocarcinoma of the pancreas-616 patients: Results, outcomes, and prognostic indicators. J. Gastrointest. Surg., 4, 567-579.

Soler, M.J., Lloveras, J. \& Batlle, D. (2008) Angiotensin converting enzyme 2 and its emerging role in the regulation of the renin angiotensin system. Med. Clin., 131, 230-236.

Sugimoto, M., Furuta, T., Shirai, N., Ikuma, M., Sugimura, H. \& Hishida, A. (2006) Influences of chymase and angiotensin I-converting enzyme gene polymorphisms on gastric cancer risks in japan. Cancer Epidemiol. Biomarkers Prev., 15, 1929-1934.

Uemura, H., Ishiguro, H., Nakaigawa, N., Nagashima, Y., Miyoshi, Y., Fujinami, K., Sakaguchi, A. \& Kubota, Y. (2003) Angiotensin II receptor blocker shows antiproliferative activity in prostate cancer cells: A possibility of tyrosine kinase inhibitor of growth factor. Mol. Cancer Therapeu., 2, 1139-1147. 
Wakahara, S., Konoshita, T., Mizuno, S., Motomura, M., Aoyama, C., Makino, Y., Kato, N., Koni, I. \& Miyamori, I. (2007) Synergistic expression of angiotensin-converting enzyme (ACE) and ACE2 in human renal tissue and confounding effects of hypertension on the ace to ace 2 ratio. Endocrinology, 148, 2453-2457.

Wang, G., Lai, F.M., Lai, K.B., Chow, K.M., Kwan, C.H., Li, K.T. \& Szeto, C.C. (2008) Urinary mrna expression of ACE and
ACE2 in human type 2 diabetic nephropathy. Diabetologia, 51, 1062-1067.

Yamamoto, K., Ohishi, M., Katsuya, T., Ito, N., Ikushima, M., Kaibe, M., Tatara, Y., Shiota, A., Sugano, S., Takeda, S., Rakugi, H. \& Ogihara, T. (2006) Deletion of angiotensin-converting enzyme 2 accelerates pressure overload-induced cardiac dysfunction by increasing local angiotensin II. Hypertension, 47, 718-726. 